\title{
Image formation in coronagraphs due to mirror polarization aberrations
}

\section{Russell A. Chipman}

Russell A. Chipman, "Image formation in coronagraphs due to mirror polarization aberrations," Proc. SPIE 10590, International Optical Design Conference 2017, 105901V (27 November 2017); doi: 10.1117/12.2291913

SPIE Event: International Optical Design Conference - IODC 2017, 2017, Denver, United States 


\title{
Image Formation in Coronagraphs Due To Mirror Polarization Aberrations
}

\author{
Russell A. Chipman
}

University of Arizona College of Optical Sciences, 1630 E. University Blvd, Tucson, AZ 85721

\begin{abstract}
The PSF for astronomical telescopes and instruments depends not only on geometric wavefront aberrations, but also on those polarization aberrations from the polarization properties of reflecting and transmitting surfaces. The image plane irradiance distribution is the linear superposition of four PSF images: one for each of the two orthogonal polarizations and one for each of two cross-coupled polarization terms.
\end{abstract}

Keywords: Polarization aberration

\section{INTRODUCTION}

The following is the reformatted conference poster presentation.

Exoplanet coronagraphs have the most demanding specifications on image quality. Next generation coronagraphs seek to image planets $<10^{-10}$ of a star brightness within a few $\lambda / \mathrm{d}$. The PSF for astronomical telescopes and instruments depends not only on geometric wavefront aberrations, but also on those polarization aberrations from the polarization properties of reflecting and transmitting surfaces. The image plane irradiance distribution is the linear superposition of four PSF images: one for each of the two orthogonal polarizations and one for each of two cross-coupled polarization terms. Coating polarization effects can interfere with coronagraph contrast if not controlled.

\section{MIRROR COATINGS, TYPICAL BEHAVIORS}

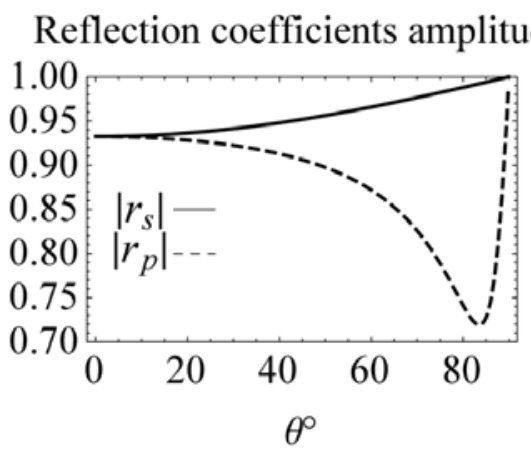

(a)

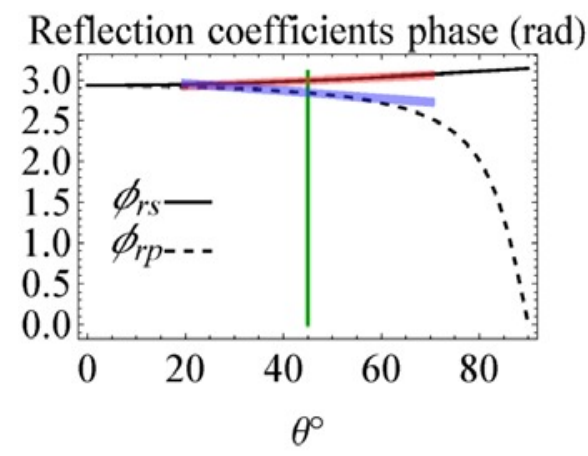

Figure 1. Example coating. Aluminum coating polarized reflectance. 


\section{SECOND ORDER POLARIZATION ABERRATION EXPANSION}

$$
\begin{aligned}
\mathbf{J} & =\boldsymbol{\sigma}_{0}+\boldsymbol{\sigma}_{1}\left[\frac{\left(d_{0}+i \Delta_{0}\right)-\left(d_{1}+i \Delta_{1}\right) \rho \sin \phi+\left(d_{2}+i \Delta_{2}\right) \rho \cos 2 \phi}{2}\right] \\
& +\sigma_{2} \frac{\left(d_{1}+i \Delta_{1}\right) \rho \cos \phi+\left(d_{2}+i \Delta_{2}\right) \rho^{2} \sin 2 \phi}{2} .
\end{aligned}
$$

Pauli matrices,

$$
\boldsymbol{\sigma}_{0}=\left(\begin{array}{ll}
1 & 0 \\
0 & 1
\end{array}\right), \quad \boldsymbol{\sigma}_{1}=\left(\begin{array}{cc}
1 & 0 \\
0 & -1
\end{array}\right), \quad \boldsymbol{\sigma}_{2}=\left(\begin{array}{ll}
0 & 1 \\
1 & 0
\end{array}\right), \quad \boldsymbol{\sigma}_{3}=\left(\begin{array}{ll}
0 & -i \\
i & 0
\end{array}\right)
$$
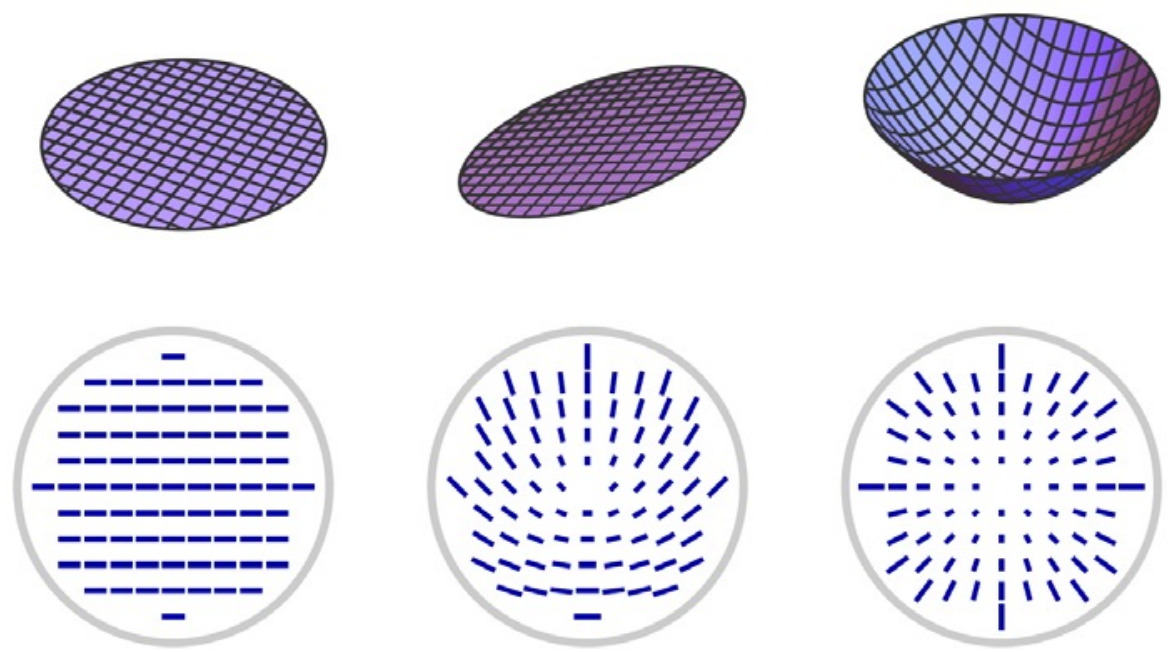

Figure 2. Second order polarization aberration expansion terms.

\section{ABERRATION EXPRESSION FOR THE JONES PUPIL}

The Jones pupil expresses the wavefront and polarization aberration as a Jones matrix function. Ideal Jones pupil is the identity matrix.

$$
\mathbf{E}^{\prime}=\left(\begin{array}{ll}
j_{x x} & j_{x y} \\
j_{y x} & j_{y y}
\end{array}\right)\left(\begin{array}{l}
E_{x} \\
E_{y}
\end{array}\right)=\left(\begin{array}{l}
E_{x}^{\prime} \\
E_{y}^{\prime}
\end{array}\right) .
$$

Example coronagraph Jones pupil determined from polarization ray tracing. 

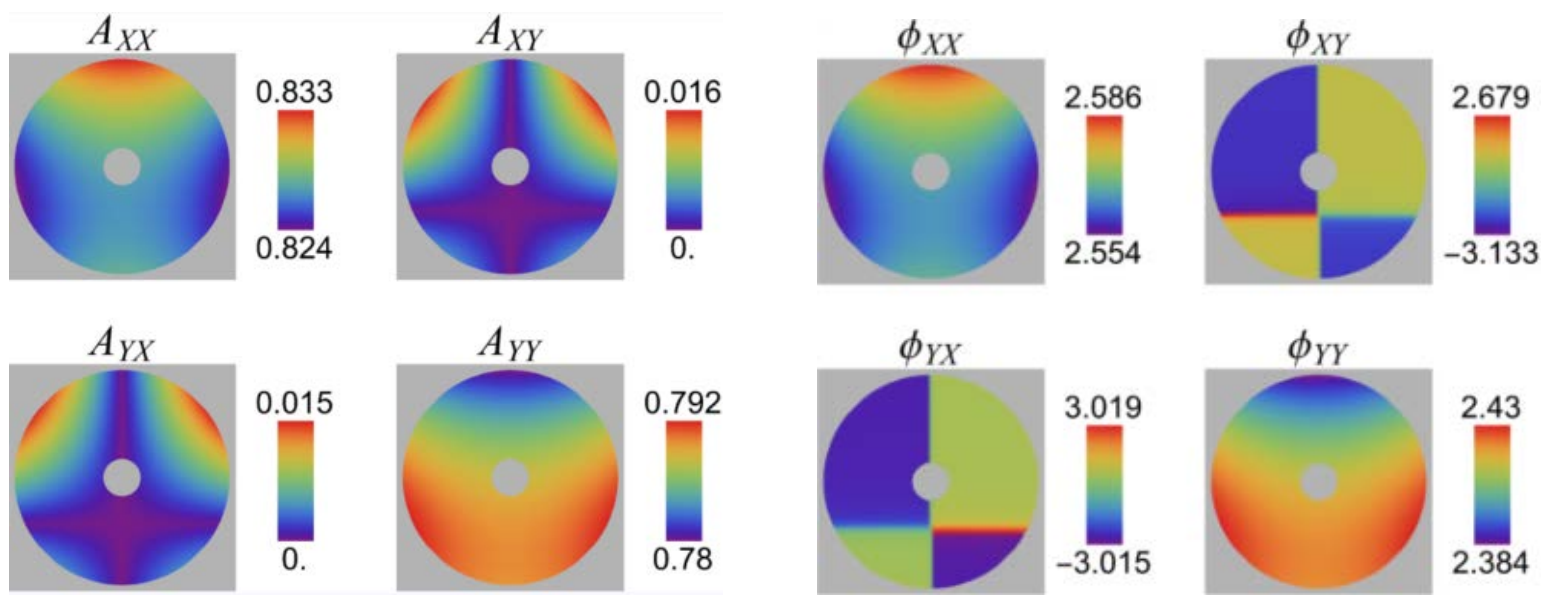

Figure 3. Amplitude.

Phase.

This Jones pupil is calculated from Polaris-M polarization ray trace (Airy Optics, Inc.) The Jones pupil is close to the identity matrix, the coating-induced polarization aberrations are small. Deviations from the identity matrix are due to the mirror's diattenuation and retardance.

Amplitudes $A_{X X}$ and $A_{Y Y}$ are constant to within $2 \%$ with small apodization. Since $A_{X Y}$ and $A_{X Y}$ are highly apodized, their diffraction patterns are significantly larger than images associated with the diagonal terms. Phases $\phi_{X Y}$ and $\phi_{Y y}$. Are polarization dependent wavefront aberrations with opposite vertical tilts, retardance tilt. The polarization aberrations couple small parts of each polarization component of the light $\left(\sim 10^{-4}\right)$ into the orthogonal polarization where these components cause highly distorted secondary, or "ghost" PSF images. The amplitude changing sign across the $\mathrm{x}$ and $\mathrm{y}$-axes causes the phase discontinuities in $\phi_{X Y}$ and $\phi_{Y X}$.

\section{CATALOG OF SECOND ORDER POLARIZATION ABERRATIONS}

Diattenuation aberrations - polarization dependent transmission aberrations from s and p-reflection differences.

Retardance aberrations - polarization dependent wavefront aberrations from s and p-phase differences. 


\subsection{Retardance defocus}

- On-axis

- Polarization-dependent astigmatism

- Unpolarized PSF is radially symmetric but broadened

- $\quad$ Cross-polarized PSF has 4 islands

$$
\mathbf{J}_{\text {RDefocus }}=\sigma_{0}+\frac{i \Delta_{2} \rho^{2}}{2}\left(\sigma_{1} \cos 2 \phi+\sigma_{2} \sin 2 \phi\right)
$$

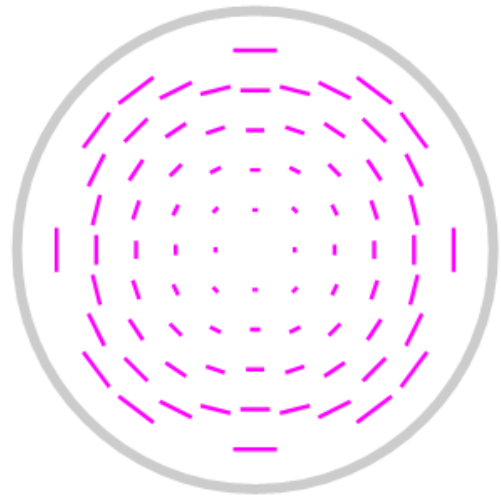

Figure 4. Retardance map
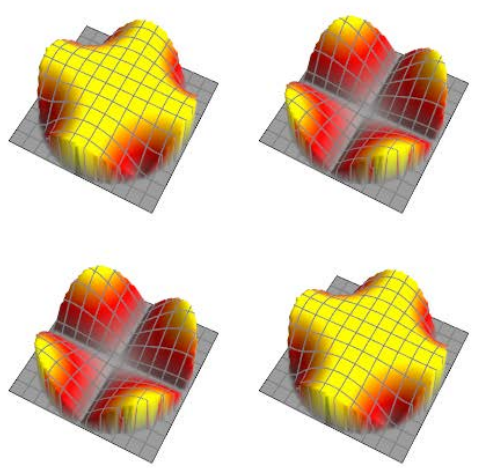

Figure 5. Jones pupil amplitude.
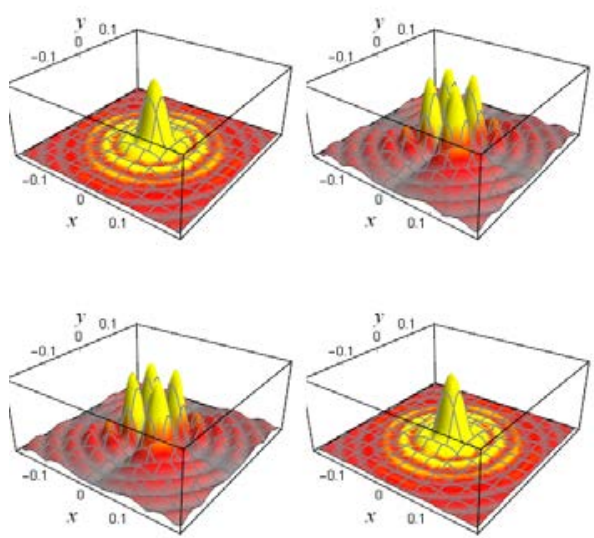

Figure 6. Amplitude Response Function Sqrt[PSF]

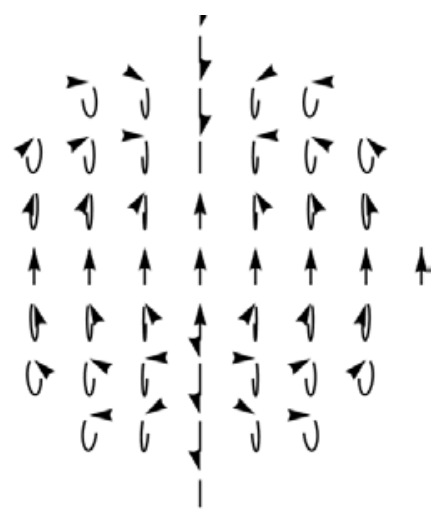

Polarization Ellipses
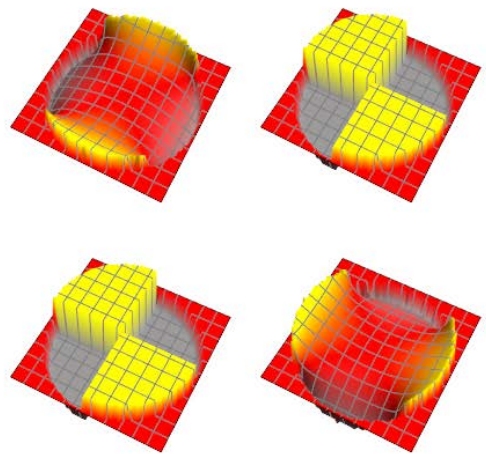

Phase. 


\subsection{Retardance tilt}

- On-axis

- Polarization-dependent astigmatism

- Unpolarized PSF is radially symmetric but broadened

- $\quad$ Cross-polarized PSF has 4 islands

$$
\mathbf{J}_{\text {RTilt }}=\sigma_{0}+\frac{i \Delta_{1} \rho}{2}\left(-\sigma_{1} \sin \phi+\sigma_{2} \cos \phi\right)
$$

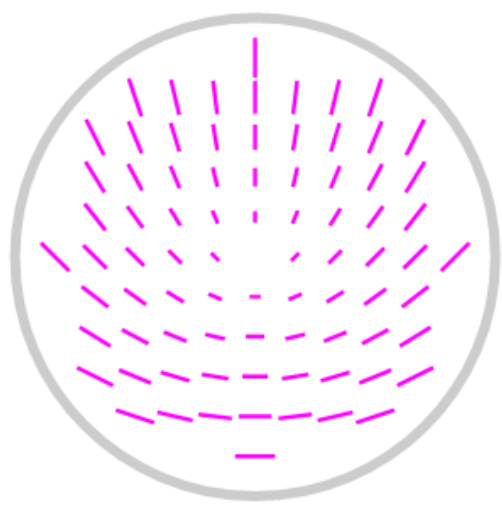

Figure 7. Retardance map
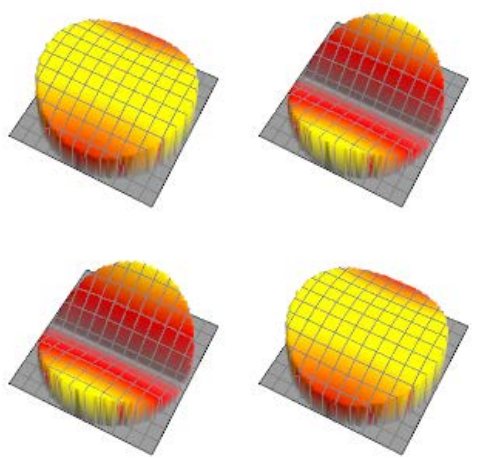

Figure 8. Jones pupil amplitude.
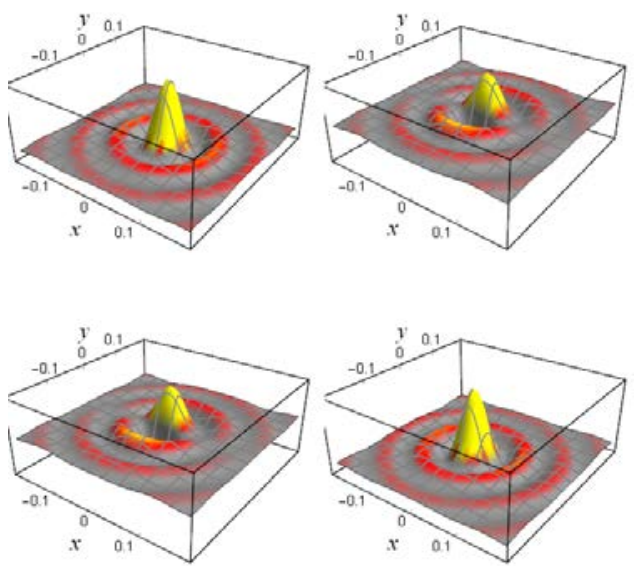

Figure 9. Amplitude Response Function Sqrt[PSF]
Polarization Ellipses
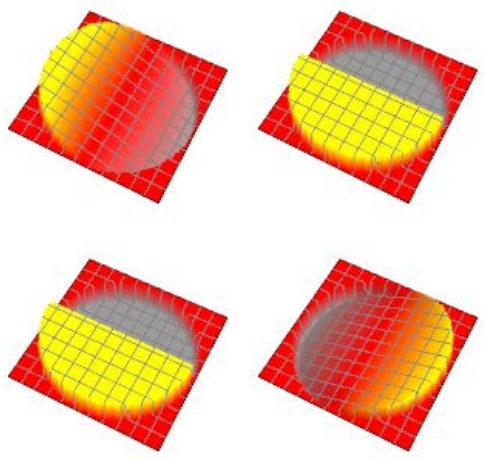

Phase. 


\subsection{Diattenuation defocus}

- On-axis

- Crossed polarized pupils have Maltese cross

- Unpolarized PSF is radially symmetric but broadened

- $\quad$ Cross-polarized PSF has 4 islands

$$
\mathbf{J}_{\text {DiDefocus }}=\sigma_{0}+\frac{d_{2} \rho^{2}}{2}\left(\sigma_{1} \cos 2 \phi+\sigma_{2} \sin 2 \phi\right)
$$

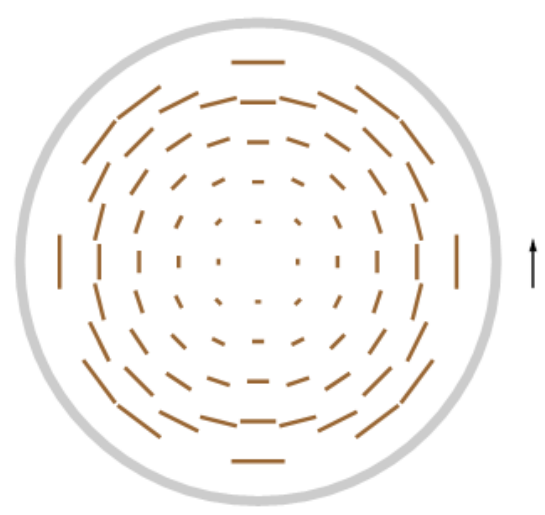

Figure9. Diattenuation map
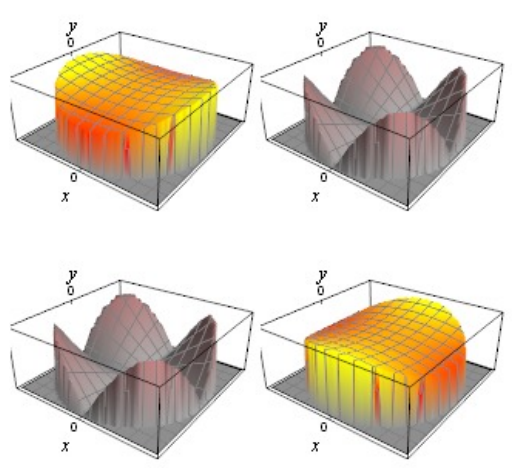

Figure 10. Jones pupil amplitude.

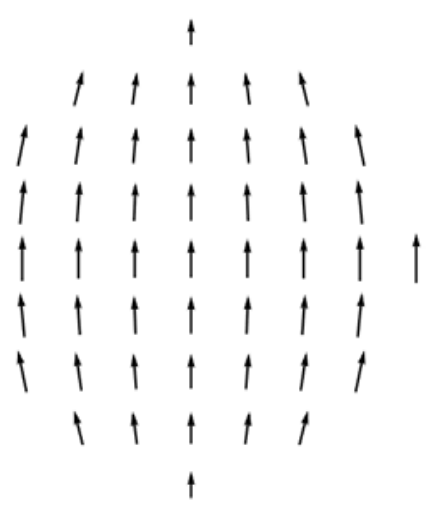

Polarization Ellipses
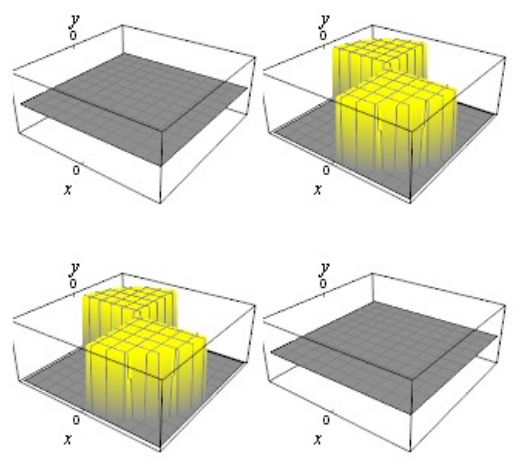

Phase.
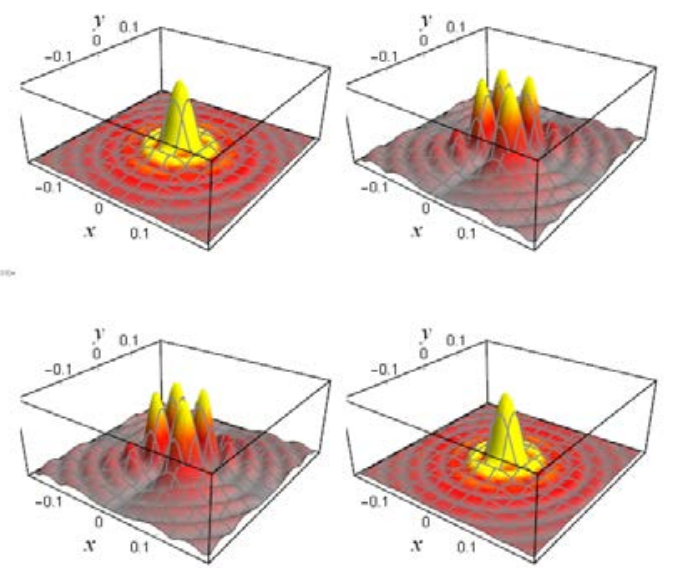

Figure 11. Amplitude Response Function Sqrt[PSF] 


\subsection{Diattenuation tilt}

- Linear in field

- $\quad$ Apodizes top of x-pupil, bottom of y-pupil

- $\quad$ Stretches co-polarized PSF components

$$
\mathbf{J}_{\text {DiTilt }}=\sigma_{0}+\frac{d_{1} \rho}{2}\left(-\sigma_{1} \sin \phi+\sigma_{2} \cos \phi\right)
$$

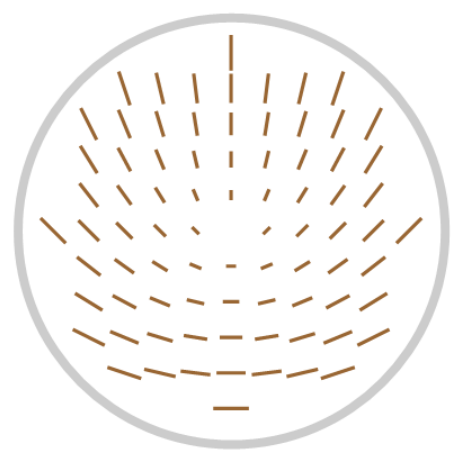

Figure 12. Diattenuation map
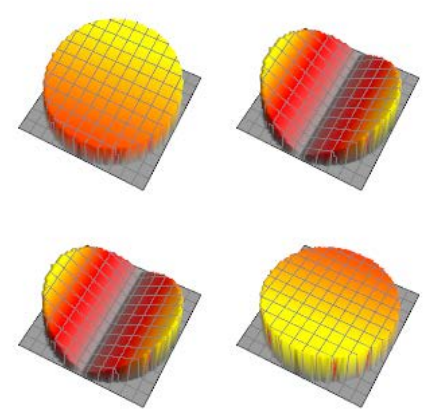

Figure 13. Jones pupil amplitude.
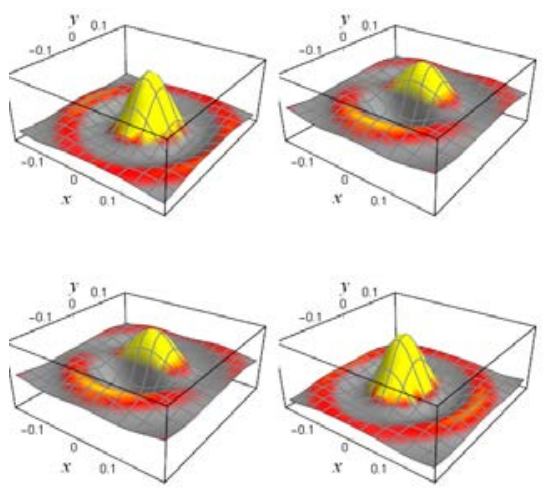

Figure 14. Amplitude Response Function Sqrt[PSF]

\section{REFERENCES}

[1] Breckinridge, James B., Wai Sze T. Lam, and Russell A. Chipman. "Polarization aberrations in astronomical telescopes: the point spread function." Publications of the Astronomical Society of the Pacific 127.951 (2015): 445. 